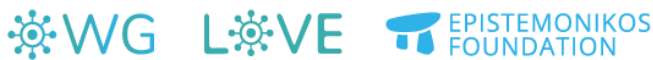

\section{Angiotensin-converting-enzyme inhibitors and angiotensin II receptor blockers for COVID-19: A living systematic review protocol}

AUTHORS:

\section{Nicolás Meza}

ORCID: 0000-0001-9505-0358

Interdisciplinary Centre for Health Studies (CIESAL), Universidad de Valparaíso, Cochrane Chile Associated Centre, Viña del Mar, Chile

\section{Javier Pérez-Bracchiglione} ORCID: 0000-0001-8738-2184

Interdisciplinary Centre for Health Studies (CIESAL), Universidad de Valparaíso, Cochrane Chile Associated Centre, Viña del Mar, Chile

\section{Ignacio Pérez}

ORCID: 0000-0002-2063-2537

School of Medicine, Cochrane Chile Associated Centre, Universidad de Valparaíso, Viña del Mar, Chile.

\section{Cristhian Carvajal}

ORCID: 0000-0001-7553-7361

School of Medicine, Cochrane Chile Associated Centre, Universidad de Valparaíso, Viña del Mar, Chile.

\section{Pablo Olguín}

ORCID: 0000-0002-0740-1128

School of Medicine, Cochrane Chile Associated Centre, Universidad de Valparaíso, Viña del Mar, Chile.

\section{Gabriel Rada}

ORCID: 0000-0003-2435-0710

Fundación Epistemonikos, Santiago, Chile

UC Evidence Center, Cochrane Chile Associated Center, Pontificia Universidad Católica de Chile, Santiago, Chile

Internal Medicine Department, Faculty of Medicine, Pontificia Universidad Católica de Chile, Santiago, Chile

\section{Eva Madrid}

ORCID: 0000-0002-8095-5549

Interdisciplinary Centre for Health Studies (CIESAL), Universidad de Valparaíso, Cochrane Chile Associated Centre, Viña del Mar, Chile

\section{COVID-19 L.OVE Working Group}

\section{Corresponding author:}

Eva Madrid

Email address: eva.madrid@uv.cl

Postal address: Angamos 655 Reñaca, Viña del Mar, Chile. 


\section{ABSTRACT}

\section{Objective}

This living systematic review aims to provide a timely, rigorous and continuously updated summary of the evidence available on the role of angiotensin-converting enzyme inhibitors (ACEi) and angiotensin II receptor blockers (ARB) in the treatment of patients with COVID-19.

\section{Design}

This is the protocol of a living systematic review.

\section{Data sources}

We will conduct searches in PubMed/Medline, Embase, Cochrane Central Register of Controlled Trials (CENTRAL), grey literature and in a centralised repository in L.OVE (Living OVerview of Evidence). L.OVE is a platform that maps PICO questions to evidence from Epistemonikos database. In response to the COVID-19 emergency, L.OVE was adapted to expand the range of evidence it covers and customised to group all COVID-19 evidence in one place. The search will cover the period until the day before submission to a journal.

\section{Eligibility criteria for selecting studies and methods}

We adapted an already published common protocol for multiple parallel systematic reviews to the specificities of this question.

We will include randomised trials evaluating the effect of either suspension or indication of angiotensin-converting-enzyme inhibitors or angiotensin II receptor blockers, as monotherapy, or in combination, versus placebo or no treatment, in patients with COVID-19. Randomised trials evaluating angiotensin-converting-enzyme inhibitors or angiotensin $\|$ receptor blockers in infections caused by other coronaviruses, such as MERS-CoV and SARS-CoV, and non-randomised studies in COVID-19 will be searched in case we find no direct evidence from randomised trials, or if the direct evidence provides low- or very low-certainty for critical outcomes.

Two reviewers will independently screen each study for eligibility, extract data, and assess the risk of bias. We will pool the results using meta-analysis and will apply the GRADE system to assess the certainty of the evidence for each outcome.

A living, web-based version of this review will be openly available during the COVID-19 pandemic. We will resubmit it every time the conclusions change or whenever there are substantial updates.

\section{Ethics and dissemination}

No ethics approval is considered necessary. The results of this review will be widely disseminated via peer-reviewed publications, social networks and traditional media. 
Angiotensin-converting-enzyme and angiotensin II receptor

blockers for COVID-19: A living systematic review protocol

\section{PROSPERO registration number}

CRD42020182495

\section{Keywords}

COVID-19, severe acute respiratory syndrome coronavirus 2, Coronavirus Infections, Systematic review, angiotensin-converting enzyme inhibitors, angiotensin II receptor blockers.

\section{INTRODUCTION}

COVID-19 is an infection caused by the SARS-CoV-2 coronavirus [1]. It was first identified in Wuhan, China, on December 31, 2019 [2]; three months later, almost half a million cases of contagion had been identified across 197 countries [3]. On March 11, 2020, WHO characterised the COVID-19 outbreak as a pandemic [1].

While the majority of cases result in mild symptoms, some might progress to pneumonia, acute respiratory distress syndrome and death $[4],[5],[6]$. The case fatality rate reported across countries, settings and age groups is highly variable, but it ranges from about $0.5 \%$ to $10 \%$ [7]. In hospitalised patients it has been reported to be higher than $10 \%$ in some centres [8].

Several studies confirm that severe acute respiratory syndrome coronavirus 2 (SARS-CoV-2) similar to SARS-CoV - uses the angiotensin-converting enzyme 2 (ACE2) receptor for host cell entry. [9],[10],[11]. So, the potential role for angiotensin-converting-enzyme inhibitors (ACEi) or angiotensin II receptor blockers (ARB) has been the subject of much debate.

It has been theorised that patients with COVID-19 comorbid to cardiovascular diseases (such as diabetes or arterial hypertension) might present an ACE2 overexpression [12]. This, because it has been also thought that ARB and ACEi drugs - generally used as anti-hypertensive therapy - could cause an up-regulation effect on ACE2, leading to the possibility of severe forms of COVID-19 [12]. Indeed, some observational studies show both hypertension and diabetes mellitus as independent risk factors of mortality for patients with COVID-19 admitted to hospital [13],[14]. However, the underlying mechanisms seem to be much more complex than initially thought [15]. In fact, some authors propose that SARS-CoV-2 could down-regulate the presence of ACE2 receptors in lung, kidney and heart, triggering a harmful hyperactivation of the renin-angiotensin system $[10],[16],[17]$ just as SARS-CoV has shown [18],[19].

Thus, the therapeutic scope of ARB or ACEi in the clinical condition of patients with COVID-19 remains unclear. 
Angiotensin-converting-enzyme and angiotensin II receptor

blockers for COVID-19: A living systematic review protocol

Using innovative and agile processes, taking advantage of technological tools, and resorting to the collective effort of several research groups, this living systematic review aims to provide a timely, rigorous and continuously updated summary of the evidence available on the role of angiotensin-converting enzyme inhibitors (ACEi) and angiotensin II receptor blockers (ARB) in the treatment of patients with COVID-19.

\section{METHODS}

\section{Protocol and registration}

This manuscript complies with the 'Preferred Reporting Items for Systematic reviews and Meta-Analyses' (PRISMA) guidelines for reporting systematic reviews and meta-analyses [20].

A protocol stating the shared objectives and methodology of multiple evidence syntheses (systematic reviews and overviews of systematic reviews) to be conducted in parallel for different questions relevant to COVID-19 was published elsewhere [21]. This protocol was adapted to the specificities of the question assessed in this review and submitted to PROSPERO (ID 182495).

\section{Search strategies}

\section{Electronic searches}

Our literature search was devised by the team maintaining the L.OVE platform (https://app.iloveevidence.com), using the following approach:

1. Identification of terms relevant to the population and intervention components of the search strategy, using Word2vec technology [22] to the corpus of documents available in Epistemonikos Database.

2. Discussion of terms with content and methods experts to identify relevant, irrelevant and missing terms.

3. Creation of a sensitive boolean strategy encompassing all the relevant terms

4. Iterative analysis of articles missed by the boolean strategy, and refinement of the strategy accordingly.

Our main search source will be Epistemonikos database (https://www.epistemonikos.org), a comprehensive database of systematic reviews and other types of evidence [23] that we supplemented with articles from multiple sources relevant to COVID-19 [24]. 
Angiotensin-converting-enzyme and angiotensin II receptor

blockers for COVID-19: A living systematic review protocol

In sum, Epistemonikos Database acts as a central repository. Only articles fulfilling Epistemonikos criteria are visible by users. The remaining articles are only accessible for members of COVID-19 L.OVE Working Group.

We will conduct additional searches using highly sensitive searches in PubMed/MEDLINE, the Cochrane Central Register of Controlled Trials (CENTRAL), Embase and the WHO International Clinical Trials Registry Platform.

The searches will cover from the inception date of each database until the day before submission. No study design, publication status or language restriction will be applied to the searches in Epistemonikos or the additional searches.

The following strategy will be used to search in Epistemonikos Database. We will adapt it to the syntax of other databases.

(coronavir* OR coronovirus* OR "corona virus" OR "virus corona" OR "corono virus" OR "virus corono" OR hcov* OR "covid-19" OR covid19* OR "covid 19" OR "2019-nCoV" OR cv19* OR "cv-19" OR "cv 19" OR "n-cov" OR ncov* OR "sars-cov-2" OR "sars-cov2" OR "SARS-Coronavirus-2" OR "SARS-Coronavirus2" OR (wuhan* AND (virus OR viruses OR viral)) OR (covid* AND (virus OR viruses OR viral)) OR "sars-cov" OR "sars cov" OR "sars-coronavirus" OR "severe acute respiratory syndrome" OR "mers-cov" OR "mers cov" OR "middle east respiratory syndrome" OR "middle-east respiratory syndrome" OR "covid-19-related" OR "SARS-CoV-2-related" OR

"SARS-CoV2-related" OR "2019-nCoV-related" OR "cv-19-related" OR "n-cov-related") AND ( (("renin-angiotensin" OR "renin angiotensin" OR (renin* AND angiotensin*) OR "renin-angiotensin-aldosterone") AND (inhibit* OR block* OR antag* OR anti)) OR RAAS OR RAAB) OR (("angiotensin-converting" OR (angiotensin* AND converting*) OR ACE OR "angiotensin-converting-enzyme") AND (inhibit* OR block* OR antag* OR anti)) OR aceis* OR "ace-inhibitor" OR "ace-inhibitors" OR "ace-i" OR "ace-is" OR (captopril* OR Capoten*) OR (enalapril* OR Vasotec* OR Renitec* OR Enacard*) OR (lisinopril* OR Prinivil* OR Zestril*) OR (perindopril* OR Coversyl* OR Coversum* OR Aceon*) OR (ramipril* OR Altace*) OR (quinapril* OR Accupril*) OR (benazepril* OR Lotensin*) OR cilazapril* OR (fosinopril* OR Monopril*) OR (trandolapril* OR Mavik*) OR (spirapril* OR Renormax*) OR (delapril* OR alindapril*) OR (moexipril* OR Univasc) OR temocapril* OR (zofenopril* OR Zocardis*) OR (imidapril* OR Tanatril*) OR alacepril*) OR (("angiotensin-receptor" OR (angiotensin* AND receptor*) OR "angiotensin-ii" OR "angiotensin ii" OR "angiotensin ii-receptor") AND (inhibit* OR block* OR antag* OR anti)) OR arbs* OR "angiotensin-receptor-blocker" OR "angiotensin-receptor-blockers" OR aiira* OR (losartan* OR Cozaar*) OR (eprosartan* OR Teveten*) OR (valsartan* OR Diovan*) OR (irbesartan* OR Avapro*) OR tasosartan* OR (candesartan* OR Atacand*) OR (telmisartan* OR Micardis* OR Actavis*) OR (olmesartan* OR Benicar*) OR (azilsartan* OR Edarbi* OR Azilva* OR "TAK-536"* OR "TAK 536"* OR TAK536* OR "TAK-491"* OR "TAK 491"* OR TAK491*) OR (fimasartan* OR Kanarb*) OR abitesartan* OR elisartan* OR embusartan* OR (forasartan* OR "SC-52458" OR "SC-52458" OR SC52458*) OR milfasartan* OR saprisartan* OR zolasartan*))

\section{Other sources}

In order to identify articles that might have been missed in the electronic searches, we will do the following: 
1. Screen the reference lists of other systematic reviews and evaluate in full text all the articles they include.

2. Scan the reference lists of selected guidelines, narrative reviews and other documents.

3. Conduct cross-citation search in Google Scholar and Microsoft Academic, using each included study as the index reference.

4. Review websites specialised in COVID-19.

5. Email the contact authors of all the included studies to ask for additional publications or data on their studies, and for other studies in the topic.

6. Review the reference list of each included study.

\section{Eligibility criteria}

\section{Types of studies}

We will preferentially include randomised trials. However, information from non-randomised studies will be used if there is no direct evidence from randomised trials or the certainty of evidence for the critical outcomes resulting from the randomised trials is graded as low- or very low, and the certainty provided by the non-randomised evidence grades higher than the one provided by the randomised evidence [25].

We will exclude studies evaluating the effects on animal models or in vitro conditions.

\section{Types of participants}

We will include trials assessing participants with COVID-19, as defined by the authors of the trials.

Whenever we find substantial clinical heterogeneity on how the condition was defined, we will explore it using a sensitivity analysis.

In case we find no direct evidence from randomised trials, or if the evidence from randomised trials provides low- or very low-certainty evidence for critical outcomes, we will include information from randomised trials evaluating ACEi or ARB in other coronavirus infections, such as MERS-CoV or SARS-CoV infections [25].

\section{Type of interventions}

The interventions of interest are ACEi or ARB. We will not restrict our criteria to any dosage, duration, timing or route of administration.

The comparison of interest will be placebo (ACEi or ARB plus optimal treatment versus placebo plus optimal treatment) or no treatment (ACEi or ARB plus optimal treatment versus optimal treatment). 
Angiotensin-converting-enzyme and angiotensin II receptor

blockers for COVID-19: A living systematic review protocol

Trials evaluating ACEi or ARB in combination versus placebo ((ACEi plus ARB plus optimal treatment versus placebo plus optimal treatment) or no treatment (ACEi plus ARB plus optimal treatment versus optimal treatment) will be also included.

Trials assessing ACEi or ARB suspension will be also included.

Trials assessing ACEi or ARB or ACEi/ARB combination plus other drugs will be eligible if the cointerventions are identical in both intervention and comparison groups.

\section{Type of outcomes}

We will not use the outcomes as an inclusion criteria during the selection process. Any article meeting all the criteria except for the outcome criterion will be preliminarily included and assessed in full text.

We used the core outcome set COS-COVID [26], the existing guidelines and reviews and the judgement of the authors of this review as an input for selecting the primary and secondary outcomes, as well as to decide upon inclusion. The review team will revise this list of outcomes, in order to incorporate ongoing efforts to define Core Outcomes Sets (e.g. COVID-19 Core Outcomes [27].

Primary outcome

- All-cause mortality

Secondary outcomes

- Mechanical ventilation

- Extracorporeal membrane oxygenation

- Length of hospital stay

- Respiratory failure

- Serious adverse events

- Time to SARS-CoV-2 RT-PCR negativity

Other outcomes

- Acute respiratory distress syndrome

- Total adverse events 
Angiotensin-converting-enzyme and angiotensin II receptor

Primary and secondary outcomes will be presented in the GRADE 'Summary of Findings' tables, and a table with all the outcomes will be presented as an appendix [28].

\section{Selection of studies}

The results of the literature search in Epistemonikos database will be automatically incorporated into the L.OVE platform (automated retrieval), where they will be de-duplicated by an algorithm comparing unique identifiers (database ID, DOI, trial registry ID), and citation details (i.e. author names, journal, year of publication, volume, number, pages, article title and article abstract). The additional searches will be uploaded to the screening software Collaboratron ${ }^{\mathrm{TM}}$ [29].

In both L.OVE platform and Collaboratron ${ }^{T M}$, two researchers will independently screen the titles and abstracts yielded by the search against the inclusion criteria. We will obtain the full reports for all titles that appear to meet the inclusion criteria or require further analysis to decide about their inclusion.

We will record the reasons for excluding trials in any stage of the search and outline the study selection process in a PRISMA flow diagram adapted for the purpose of this project .

\section{Extraction and management of data}

Using standardised forms, two reviewers will independently extract data from each included study. We will collect the following information: study design, setting, participant characteristics (including disease severity and age) and study eligibility criteria; details about the administered intervention and comparison, including dose and therapeutic scheme, duration, timing (i.e. time after diagnosis) and route of administration; the outcomes assessed and the time they were measured; the source of funding of the study and the conflicts of interest disclosed by the investigators; the risk of bias assessment for each individual study.

We will resolve disagreements by discussion, and one arbiter will adjudicate unresolved disagreements.

\section{Risk of bias assessment}

The risk of bias for each randomised trial will be assessed using the 'risk of bias' tool (RoB 2.0: a revised tool to assess risk of bias in randomised trials) [30]. We will consider the effect of assignment to the intervention for this review. Two reviewers will independently assess five 
Angiotensin-converting-enzyme and angiotensin II receptor

blockers for COVID-19: A living systematic review protocol

domains of bias for each outcome result of all reported outcomes and time points. These five domains are: bias due to (1) the randomisation process, (2) deviations from intended interventions (effects of assignment to interventions at baseline), (3) missing outcome data, (4) measurement of the outcome, and (5) selection of reported results. Answers to signalling questions and collectively supporting information will lead to a domain-level judgement in the form of 'Low risk of bias', 'Some concerns', or 'High risk of bias'. These domain-level judgements will inform an overall 'risk of bias' judgement for each result. Discrepancies between review authors will be resolved by discussion to reach consensus. If necessary, a third review author will be consulted to achieve a decision.

We will assess the risks of bias of other study designs with the ROBINS-I tool (ROBINS-I: Risk Of Bias In Non-randomised Studies of Interventions) [31]. We will assess the following domains: bias due to confounding, bias in selection of participants into the study, bias in classification of interventions, bias due to deviations from intended interventions (effect of assignment to intervention), bias due to missing data, bias in measurement of outcomes and bias in the selection of the reported result. We will judge each domain as low risk, moderate risk, serious risk, critical risk, or no information, and evaluate individual bias items as described in ROBINS-I guidance. We will not consider time-varying confounding, as these confounders are not relevant in this setting [31].

We will consider the following factors as baseline potential confounders:

- Age

- Comorbidities (e.g. cardiovascular disease, renal disease, eye disease, liver disease)

- Co-interventions

- Severity, as defined by the authors (i.e respiratory failure vs respiratory distress syndrome vs ICU requirement).

\section{Measures of treatment effect}

For dichotomous outcomes, we will express the estimate of treatment effect of an intervention as risk ratios (RR) or odds ratios (OR) along with 95\% confidence intervals ( $\mathrm{Cl}$ ).

For continuous outcomes, we will use mean difference and standard deviation (SD) to summarise the data using a $95 \% \mathrm{Cl}$. Whenever continuous outcomes are measured using different scales, the treatment effect will be expressed as a standardised mean difference (SMD) with 95\% Cl. When possible, we will multiply the SMD by a standard deviation that is representative from the pooled studies, for example, the SD from a well-known scale used by several of the studies included in the analysis on which the result is based. In cases where the minimally important difference (MID) is 
Angiotensin-converting-enzyme and angiotensin II receptor

blockers for COVID-19: A living systematic review protocol

known, we will also present continuous outcomes as MID units or inform the results as the difference in the proportion of patients achieving a minimal important effect between intervention and control[32].

Then, these results will be displayed on the 'Summary of Findings Table' as mean difference [32].

\section{Strategy for data synthesis}

If we include more than one trial we will conduct a formal quantitative synthesis (meta-analysis) for studies clinically homogeneous using RevMan 5 [33], using the inverse variance method with the random-effects model. For any outcomes where data were insufficient to calculate an effect estimate, a narrative synthesis will be presented, describing the studies in terms of the direction and the size of effects, and any available measure of precision.

\section{Subgroup and sensitivity analysis}

We will perform subgroup analysis according to the definition of severe COVID-19 infection (i.e respiratory failure vs respiratory distress syndrome vs ICU requirement). In case we identify significant differences between subgroups (test for interaction $<0.05$ ) we will report the results of individual subgroups separately.

We will perform sensitivity analysis excluding high risk of bias studies, and if non-randomised studies are used, excluding studies that did not report adjusted estimates. In cases where the primary analysis effect estimates and the sensitivity analysis effect estimates significantly differ we will either present the low risk of bias - adjusted sensitivity analysis estimates - or present the primary analysis estimates but downgrading the certainty of the evidence because of risk of bias.

\section{Assessment of certainty of evidence}

The certainty of the evidence for all outcomes will be judged using the Grading of Recommendations Assessment, Development and Evaluation working group methodology

(GRADE Working Group)[34], across the domains of risk of bias, consistency, directness, precision and reporting bias. Certainty will be adjudicated as high, moderate, low or very low. For the main comparisons and outcomes, we will prepare Summary of Findings (SoF) tables [35],[32] and also interactive Summary of Findings tables (http://isof.epistemonikos.org/). A SoF table with all the comparisons and outcomes will be presented as an appendix.

\section{Living evidence synthesis}


Angiotensin-converting-enzyme and angiotensin II receptor

blockers for COVID-19: A living systematic review protocol

An artificial intelligence algorithm deployed in the Coronavirus/COVID-19 topic of the L.OVE platform will provide instant notification of articles with a high likelihood to be eligible. The authors will review them, will decide upon inclusion, and will update the living web version of the review accordingly. We will consider resubmission to a journal if there is a change in the direction of the effect on the critical outcomes or a substantial modification to the certainty of the evidence.

This review is part of a larger project set up to produce multiple parallel systematic reviews relevant to COVID-19[21].

\section{NOTES}

\section{Acknowledgements}

The members of the COVID-19 L.OVE Working Group and Epistemonikos Foundation have made possible to build the systems and compile the information needed by this project. Epistemonikos is a collaborative effort, based on the ongoing volunteer work of over a thousand contributors since 2012.

\section{Roles and contributions}

GR conceived the common protocol for all the reviews being conducted by the COVID-19 L.OVE Working Group. NM, EM and GR drafted the manuscript, and all other authors contributed to it. The corresponding author is the guarantor and declares that all authors meet authorship criteria and that no other authors meeting the criteria have been omitted.

The COVID-19 L.OVE Working Group was created by Epistemonikos and a number of expert teams in order to provide decision makers with the best evidence related to COVID-19. Up-to-date information about the group and its member organisations is available here: epistemonikos.cl/working-group

\section{Competing interests}

All authors declare no financial relationships with any organisation that might have a real or perceived interest in this work. There are no other relationships or activities that might have influenced the submitted work.

\section{Funding}

This project was not commissioned by any organisation and did not receive external funding. 
Epistemonikos Foundation is providing training, support and tools at no cost for all the members of the COVID-19 L.OVE Working Group.

\section{PROSPERO registration}

CRD42020182495

\section{Ethics}

As researchers will not access information that could lead to the identification of an individual participant, obtaining ethical approval was waived.

\section{Data sharing}

All data related to the project will be available. Epistemonikos Foundation will grant access to data. 
Angiotensin-converting-enzyme and angiotensin II receptor

\section{REFERENCES}

1. World Health Organization. Director-General's remarks at the media briefing on 2019-nCoV on 11 February 2020. [Internet] World Health Organization; 2020 [Accessed May 1, 2020] Available from: https://www.who.int/dg/speeches/detail/who-director-general-s-remarks-at-the-media-briefingon-2019-ncov-on-11-february-2020

2. Hui DS, I Azhar E, Madani TA, et al. The continuing 2019-nCoV epidemic threat of novel coronaviruses to global health - The latest 2019 novel coronavirus outbreak in Wuhan, China. Int J Infect Dis. 2020 Feb;91:264-266. Available from: doi:10.1016/j.ijid.2020.01.009

3. Dong E, Du H, Gardner L. An interactive web-based dashboard to track COVID-19 in real time. Lancet Infect Dis. 2020 Feb 19 [Accessed May 1, 2020]

Available from: doi:10.1016/S1473-3099(20)30120-1

4. Guan WJ, Ni ZY, Hu Y, et al. Clinical Characteristics of Coronavirus Disease 2019 in China. N Engl J Med 2020. Available from: doi:10.1056/NEJMoa2002032

5. Tavakoli, Ahmad, Vahdat, Katayon, Keshavarz, Mohsen. Novel Coronavirus Disease 2019 (COVID-19): An Emerging Infectious Disease in the 21st Century. BPUMS. 2020;22(6):432-450. Available from: doi:10.29252/ismj.22.6.432

6. Li LQ, Huang T, Wang YQ, Wang ZP, Liang Y, Huang TB, Zhang HY, Sun WM, Wang YP. 2019 novel coronavirus patients' clinical characteristics, discharge rate and fatality rate of meta-analysis. Journal of medical virology. 2020. Available from: doi:10.1002/jmv.25757

7. Global Covid-19 Case Fatality Rates [Internet] UK: Centre for Evidence-Based Medicine [Accessed May 1, 2020]. Available from: https://www.cebm.net/covid-19/global-covid-19-case-fatality-rates/

8. Rodriguez-Morales $A J$, Cardona-Ospina JA, Gutiérrez-Ocampo $E$, et al. Clinical, laboratory and imaging features of COVID-19: A systematic review and meta-analysis. Travel medicine and infectious disease. 2020;:101623. Available from: doi:10.1016/j.tmaid.2020.101623

9. Chen Y, Guo Y, Pan Y, Zhao ZJ. Structure analysis of the receptor binding of 2019-nCoV. Biochem Biophys Res Commun. 2020 Feb 17. pii: S0006-291X(20)30339-9. doi: 10.1016/j.bbrc.2020.02.071.

10. Zhang H, Penninger JM, Li Y, Zhong N, Slutsky AS. Angiotensin-converting enzyme 2 (ACE2) as a SARS-CoV-2 receptor: molecular mechanisms and potential therapeutic target. Intensive Care Med. 2020 Apr;46(4):586-590. doi: 10.1007/s00134-020-05985-9.

11. Hoffmann M, Kleine-Weber $H$, Schroeder S, et al. SARS-CoV-2 Cell Entry Depends on ACE2 and TMPRSS2 and Is Blocked by a Clinically Proven Protease Inhibitor. Cell. 2020 Apr 16;181(2):271-280.e8. doi: 10.1016/j.cell.2020.02.052.

12. Fang L, Karakiulakis G, Roth M. Are patients with hypertension and diabetes mellitus at increased risk for COVID-19 infection? Lancet Respir Med. 2020 Apr;8(4):e21. doi: 10.1016/S2213-2600(20)30116-8. 
13. Zhou $F, Y u T$, Du R, et al. Clinical course and risk factors for mortality of adult inpatients with COVID-19 in Wuhan, China: a retrospective cohort study. Lancet. 2020 Mar 28;395(10229):1054-1062. Doi: 10.1016/S0140-6736(20)30566-3.

14. Wu C, Chen X, Cai Y, et al. Risk Factors Associated With Acute Respiratory Distress Syndrome and Death in Patients With Coronavirus Disease 2019 Pneumonia in Wuhan, China. JAMA Intern Med. 2020 Mar 13. Doi: 10.1001/jamainternmed.2020.0994.

15. Guo J, Huang Z, Lin L, Lv J. Coronavirus Disease 2019 (COVID-19) and Cardiovascular Disease: A Viewpoint on the Potential Influence of Angiotensin-Converting Enzyme Inhibitors/Angiotensin Receptor Blockers on Onset and Severity of Severe Acute Respiratory Syndrome Coronavirus 2 Infection. J Am Heart Assoc. 2020 Apr 7;9(7):e016219. doi: 10.1161/JAHA.120.016219.

16. Vaduganathan $M$, Vardeny 0 , Michel $T$, et al. Renin-Angiotensin-Aldosterone System Inhibitors in Patients with Covid-19. N Engl J Med. 2020 Mar 30. doi: 10.1056/NEJMsr2005760.

17. de Simone G, Mancusi C. Speculation is not evidence: antihypertensive therapy and COVID-19. Eur Heart J Cardiovasc Pharmacother. 2020 Apr 1. pii: pvaa021. Doi: 10.1093/ehjcvp/pvaa021.

18. Kuba $K$, Imai $Y$, Rao $S$, et al. A crucial role of angiotensin converting enzyme 2 (ACE2) in SARS coronavirus-induced lung injury. Nat Med. 2005 Aug;11(8):875-9.

19. Patel VB, Clarke N, Wang Z, et al. Angiotensin II induced proteolytic cleavage of myocardial ACE2 is mediated by TACE/ADAM-17: a positive feedback mechanism in the RAS. J Mol Cell Cardiol. 2014 Jan;66:167-76. doi: 10.1016/j.yjmcc.2013.11.017

20. Moher D, Shamseer $L$, Clarke $M$, et al. Preferred reporting items for systematic review and meta-analysis protocols (PRISMA-P) 2015 statement. Syst Rev. 2015 Jan 1;4:1. Available from: doi:10.1186/2046-4053-4-1

21. Rada G, Verdugo-Paiva F, Ávila C, Morel-Marambio M, Bravo-Jeria R, Pesce F, Madrid E, Izcovich A; COVID-19 L.OVE Working Group. Evidence synthesis relevant to COVID-19: a protocol for multiple systematic reviews and overviews of systematic reviews. Medwave 2020;20(3):e7867. Available from: doi:10.5867/medwave.2020.03.7867

22. Github repository [Internet] [Accessed May 1, 2020 Available from: https://github.com/dperezrada/keywords2vec

23. Epistemonikos Database Methods [Internet] Santiago: Epistemonikos Foundation [Accessed May 1 , 2020] Available from: https://www.epistemonikos.org/en/about_us/methods

24. Methods for the special L.OVE of Coronavirus infection [Internet] Santiago: Epistemonikos Foundation [Accessed May 1, 2020] Available from: https://app.iloveevidence.com/covid-19

25. Schünemann HJ, Cuello C, Akl EA, Mustafa RA, Meerpohl JJ, Thayer K, Morgan RL, Gartlehner G, Kunz R, Katikireddi SV, Sterne J, Higgins JP, Guyatt G; GRADE Working Group. GRADE guidelines: 18. How ROBINS-I and other tools to assess risk of bias in nonrandomized studies should be used to rate the certainty of a body of evidence. J Clin Epidemiol. 2019 Jul;111:105-114. Available from: doi:10.1016/j.jclinepi.2018.01.012 
Angiotensin-converting-enzyme and angiotensin II receptor

26. Xinyao Jin, Bo Pang, Junhua Zhang, et al. Core Outcome Set for Clinical Trials on Coronavirus Disease 2019 (COS-COVID), Engineering, 2020. Available from: doi:10.1016/j.eng.2020.03.002

27. COVID-19 Core Outcomes [Internet]. [Accessed May 1, 2020] Available from: https://www.covid-19-cos.org/

28. Guyatt GH, Oxman AD, Santesso N, et al. GRADE guidelines: 12. Preparing summary of findings tables-binary outcomes. J Clin Epidemiol 2013 Feb;66(2):158-72.

Available from: doi:10.1016/j.jclinepi.2012.01.012

29. Collaboratron [Software]. Santiago: Epistemonikos Foundation, 2017.

30. Sterne JAC, Savović J, Page MJ, Elbers RG, Blencowe NS, Boutron I, Cates CJ, Cheng HY, Corbett MS, Eldridge SM, Emberson JR, Hernán MA, Hopewell S, Hróbjartsson A, Junqueira DR, Jüni $P$, Kirkham JJ, Lasserson T, Li T, McAleenan A, Reeves BC, Shepperd S, Shrier I, Stewart LA, Tilling K, White IR, Whiting PF, Higgins JPT. RoB 2: a revised tool for assessing risk of bias in randomised trials. BMJ. 2019 Aug 28;366:14898. Available from: doi:10.1136/bmj.14898

31. Sterne JA, Hernán MA, Reeves BC, Savović J, Berkman ND, Viswanathan M, Henry D, Altman DG, Ansari MT, Boutron I, Carpenter JR, Chan AW, Churchill R, Deeks JJ, Hróbjartsson A, Kirkham J, Jüni P, Loke YK, Pigott TD, Ramsay CR, Regidor D, Rothstein HR, Sandhu L, Santaguida PL, Schünemann $H J$, Shea B, Shrier I, Tugwell P, Turner L, Valentine JC, Waddington H, Waters E, Wells GA, Whiting PF, Higgins JP. ROBINS-I: a tool for assessing risk of bias in non-randomised studies of interventions. BMJ. 2016 Oct 12;355:i4919. Available from: doi:10.1136/bmj.i4919

32. Guyatt GH, Thorlund K, Oxman AD, et al. GRADE guidelines: 13. Preparing summary of findings tables and evidence profiles-continuous outcomes. J Clin Epidemiol 2013 Feb;66(2):173-83. Available from: doi:10.1016/j.jclinepi.2012.08.001

33. Review Manager (RevMan) [Software]. Version 5.3.5 Copenhagen: The Nordic Cochrane Centre, The Cochrane Collaboration, 2014.

34. Guyatt GH, Oxman AD, Vist GE, Kunz R, Falck-Ytter Y, Alonso-Coello P, Schünemann HJ; GRADE Working Group. GRADE: an emerging consensus on rating quality of evidence and strength of recommendations. BMJ. 2008 Apr 26;336(7650):924-6. Available from: doi:10.1136/bmj.39489.470347.AD

35. Guyatt GH, Oxman AD, Santesso N, et al. GRADE guidelines: 12. Preparing summary of findings tables-binary outcomes. J Clin Epidemiol [Internet] 2013 Feb [Accessed March 26] ;66(2):158-72. Available from: doi:10.1016/j.jclinepi.2012.01.012 